\title{
Comparative Study of Cartridge Based Nucleotide Amplification Test (CBNAAT) Versus Sputum Microscopy for the Diagnosis of Pulmonary Tuberculosis in People living with HIV and its Correlation with CD4 Count
} Authors

\section{Dr Kavya S T $\mathbf{T}^{1}$, Dr Saicharan G Chitrak ${ }^{2^{*}}$, Dr Stephen Benny ${ }^{3}$, Dr Yaduraj D K ${ }^{4}$}

${ }^{1}$ Professor of Medicine, Department of Internal Medicine, Bangalore Medical College and Research Institute, Bangalore

${ }^{2,4}$ Junior Resident, Department of Internal Medicine, Bangalore Medical College and Research Institute, Bangalore

${ }^{3}$ Senior Resident, Department of Internal Medicine, Bangalore Medical College and Research Institute, Bangalore

*Corresponding Author

Dr Saicharan G Chitrak

Junior Resident, Department of Internal Medicine, Bangalore Medical College and Research Institute,

Bangalore, India

Abstract
Background: Diagnosis of tuberculosis is a challenge on its own, more so in HIV-infected individuals.
The Xpert MTB/RIF assay has recently replaced sputum microscopy as the investigation of choice in
People living with HIV (PLHIV). The present study aims to compare two methods for the diagnosis of TB
in PLHIV: Microscopy, and Xpert MTB/RIF. and to compare with CD4 count
Objective of the Study
1. To compare between efficacy of CBNAAT and sputum microscopy in detection of pulmonary
tuberculosis in HIV patients.
2. To detect the prevalence of rifampicin resistance and MDR TB in HIV patients
Methods: Study was conducted on HIV patients consulted at hospitals attached to Bangalore medical
college and research institute, during the period of November 1st 2018 to August 30th 2020 were taken
for study, considering the inclusion and exclusion criteria. Detailed clinical history, examination and
investigations viz, sputum microscopy, CBNAAT,CD4 count was done for every patients.
Results: After analysis of the data it was found that CBNAAT was more effective in detecting
tuberculosis in PLHIV, when compared to conventional sputum microscopy. Sensitivity of CBNAAT was
positively correlated with low CD4 count. The prevalence of rifampicin resistance was $11 \%$
Conclusion: In conclusion, when used as a routine screening test among people living with HIV, rapid
screening using the Xpert MTB/RIF assay substantially increased case detection, supporting replacement
of microscopy as the initial diagnostic tool. The assay also greatly decreased the time to diagnosis of
tuberculosis and aids in early identification of Rifampicin-resistant tuberculosis.

\section{Introduction}

Tuberculosis (TB) continues to be a leading cause of morbidity and mortality and is currently the fourth most common cause of death from communicable diseases worldwide .There are about 9 million cases of newly detected 
tuberculosis every year all over the world. Out of which $24 \%$ of total TB burden is in India. Tuberculosis is the most common opportunistic infection in HIV in India. Prevalence of TB in HIV patients in India is around $17 \%$. ${ }^{(1)}$

Conventionally sputum microscopy used to detect tuberculosis in India. CBNAAT is a newer technique of real-time PCR used for tuberculosis detection and rifampicin resistance. It was in 2010. WHO first introduced CBNAAT. In India RNTCP program introduced CBNAAT in 2013.

MDR tuberculosis is defined as tuberculosis resistant to isoniazide and rifampicin. Only $28 \%$ of MDR TB is detected in 2013 because of the lack of appropriate facilities in most of the world. ${ }^{(2)}$ CBNAAT also known as GeneXpert simultaneously detect $\mathrm{TB}$ and rifampicin resistance in $<2$ hours with minimal biosafety and training requirement.

It is necessary to detect pulmonary TB in HIV patients as early possible as it is a common cause of morbidity and mortality in HIV patients. So it is high time to select a test which can detect pulmonary TB in HIV patients rapidly and with maximum reliability.

In developing countries like India, availability of test and cost effectiveness should be taken into consideration. Although sputum culture considered to be gold standard in pulmonary tuberculosis, it takes more time for the culture results making treatment to be delayed. Another problem in HIV patients with pulmonary tuberculosis is the amount of sputum produced is less as caseation is less. Concentration of bacilli in sputum of HIV patients with pulmonary TB is less.

\section{Objective of the Study}

1. To compare between efficacy of CBNAAT and sputum microscopy in detection of pulmonary tuberculosis in HIV patients.

2. To detect the prevalence of rifampicin resistance and MDR TB in HIV patients

\section{Methodology}

Source of Data: Samples were taken from patients attending ICTC and ART centres of all teaching hospitals attached to Bangalore Medical College \& Research Institute, Bangalore.

\section{Method of Collection of Data}

A. Design of Study: This was a cross sectional study in which 200 HIV-infected patients with suspected TB were included.

B. Study Period: November 1st 2018 to August 30th 2020

C. Place of study: For Xpert MTB/RIF assay, the GeneXpert installed at RNTCP, Bowring \& Lady Curzon Hospital and Victoria hospital, Bangalore was used

D. Sample Size: 200 samples.

E. Inclusion Criteria: Patients of age 18 years and over, with positive HIV status ascertained according to the NACO guidelines were screened according to the 4 symptom complex for tuberculosis (current cough, fever, weight loss, night sweats). Patients positive for one or more symptoms were included in the study.

\section{F. Exclusion Criteria:}

Patients receiving ATT at the time of enrollment

Patients having malignancy

\section{G. Methodology:}

\section{Ethics Statement}

Written informed consent was obtained from all study participants. Approval for conducting the study was taken from Karnataka State AIDS Prevention Society.

\section{Patient Evaluation}

Patients were evaluated at the time of enrolment and two sputum samples- one spot and one early morning sample were collected. The specimens collected were kept in the refrigerator until processing and all the specimens were processed within 48 hours of collection.

\section{Sputum Smear Microscopy}

Direct smears were prepared from early morning and spot sputum specimens, stained using the Ziehl-Neelsen technique and examined under the light microscope. Patients were considered smear- 
positive if at least one acid fast bacillus was seen under high power field in at least one sample.

\section{Sputum CBNAAT}

The spot sputum sample (unprocessed) was utilized for the Xpert MTB/RIF assay using the GeneXpert MTB/RIF version G4 (Cepheid, Sunnyvale, CA) installed at the RNTCP, Bowring \& Lady Curzon Hospital and Victoria Hospital Bangalore.

The sputum samples were treated with a sample reagent (SR) containing sodium hydroxide and isopropanol. The SR was added to the sample in a ratio of $2: 1$ and incubated at room temperature for $15 \mathrm{~min}$. The treated sample is then manually transferred to the cartridge which is loaded into the GeneXpert instrument. A printable test result was obtained after 1hour 45 minutes.

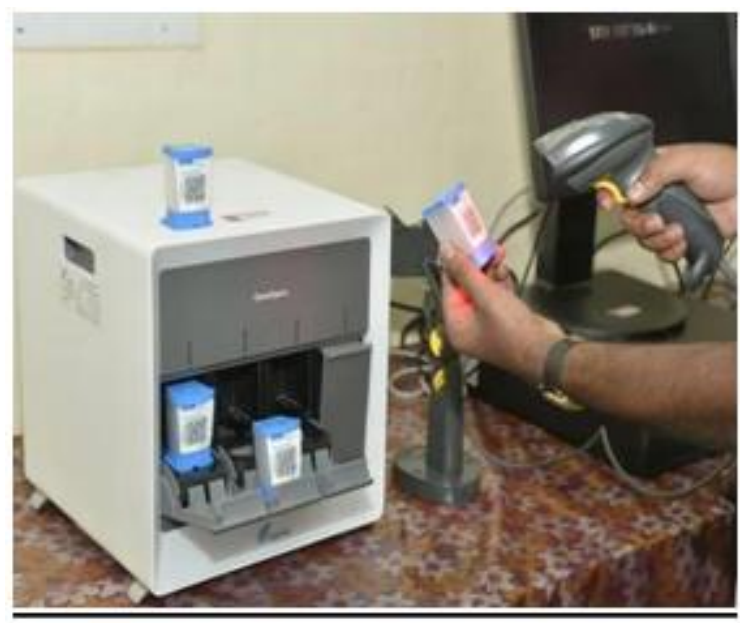

Figure 2: CBNAAT machine in B\&LCH

\section{Results}

\section{Statistical Analysis}

Data was entered into Microsoft excel data sheet and was analyzed using IBM SPSS 20 version software. Categorical data was represented in the form of Frequencies and proportions. Continuous data was represented as mean and standard deviation. Independent sample $\mathbf{t}$ test was used as test of significance to identify the mean difference between two quantitative variables. Chi square test was done to find the correlation between two grouped variables

Graphical representation of data: MS Excel and MS word was used to obtain various types of graphs such as bar diagram, Pie diagram and Scatter plots.

p value (Probability that the result is true) of $<0.05$ was considered as statistically significant after assuming all the rules of statistical tests.

Statistical Software: MS Excel, SPSS version 20 (IBM SPSS Statistics, Somers NY, USA) was used to analyze data

\section{Results and Analysis}

Total 200 patients were taken for the study.

All cases were evaluated with the help of clinical history detailed physical examination and investigations.

Study Design: A cross sectional study Age distribution of subjects in the study

Table 4-Table showing age distribution

\begin{tabular}{|l|c|c|}
\hline Age & Frequency & Percentage \\
\hline $10-20$ & 8 & 4 \\
\hline $21-30$ & 37 & 18.5 \\
\hline $31-40$ & 76 & 38 \\
\hline $41-50$ & 41 & 20.5 \\
\hline $51-60$ & 19 & 9.5 \\
\hline $61-70$ & 14 & 7 \\
\hline$>70$ & 5 & 2.5 \\
\hline
\end{tabular}

Average age of the patients was $39.4 \pm 9$ years

Figure 3 Graph showing distribution of age

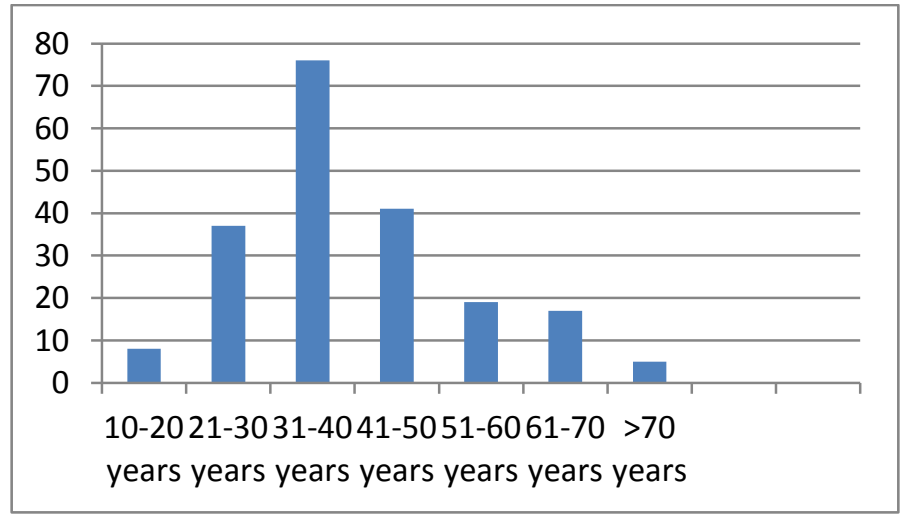

\section{Gender distribution of patients}

Table 5-Table showing gender distribution

\begin{tabular}{|c|c|c|}
\hline & Frequency & Percentage \\
\hline Male & 118 & 59 \\
\hline Female & 82 & 41 \\
\hline
\end{tabular}


Figure 4 Graph showing gender distribution

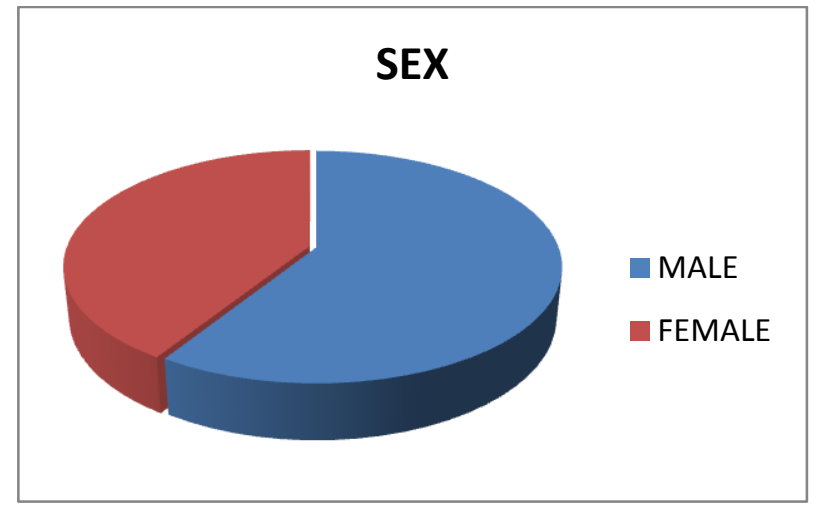

\section{Frequency of Sputum Microscopy}

Table 6- Frequency of Sputum microscopy

\begin{tabular}{|l|c|c|}
\hline Sputum microscopy & frequency & percentage \\
\hline Positive(1+) & 6 & 3 \\
\hline Positive(2+) & 1 & 0.5 \\
\hline negative & 193 & 96.5 \\
\hline
\end{tabular}

Figure 5- Pie chart showing distribution of sputum microscopy

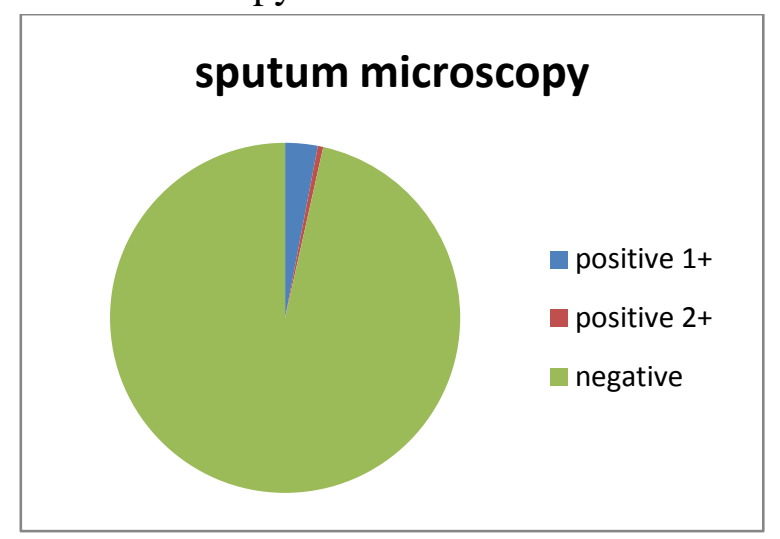

\section{Distribution of sputum CBNAAT}

Table 7-Distribution of CBNAAT

\begin{tabular}{|l|c|c|}
\hline CBNAAT & Frequency & Percentage \\
\hline Positive & 27 & 13.5 \\
\hline Negative & 173 & 86.5 \\
\hline
\end{tabular}

Figure 6- Graph showing distribution of CBNAAT

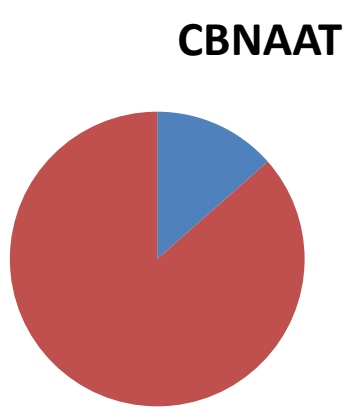

- POSITIVE

nEGATIVE

\section{Distribution of rifampicin resistance}

Table 8- distribution of rifampicin resistance

\begin{tabular}{|l|c|c|}
\hline & Frequency & Percentage \\
\hline Rif sensitive & 24 & 12 \\
\hline Rif resistant & 3 & 1.5 \\
\hline Rif not analyzed & 173 & 86.5 \\
\hline
\end{tabular}

Figure 7- Distribution of rifampicin resistance

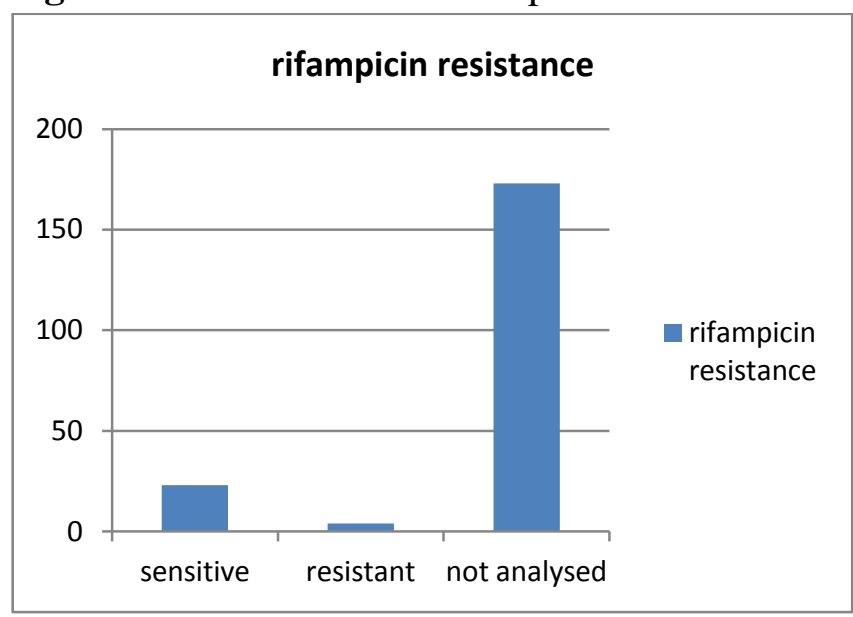

Distribution of Consolidation in Chest X-ray

Table 9 Distribution of consolidation in X ray

\begin{tabular}{|l|c|c|}
\hline X ray & frequency & percentage \\
\hline consolidation & 14 & 7 \\
\hline No consolidation & 186 & 93 \\
\hline
\end{tabular}

Figure 8 Graph showing distribution of consolidation

\section{consolidation}

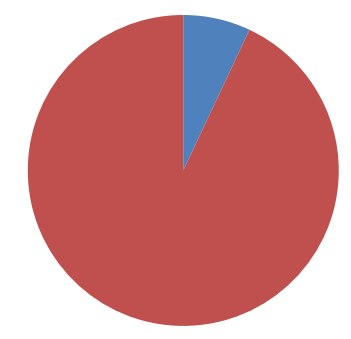

consolidation

no consolidation

Comparison between CBNAAT and sputum microscopy

Table 10- Table Comparing CBNAAT V/S Sputum microscopy

\begin{tabular}{|l|l|c|c|c|}
\hline \multicolumn{2}{|c|}{} & \multicolumn{2}{|c|}{ sputum microscopy } & \multirow{2}{*}{ Total } \\
\cline { 3 - 4 } \multicolumn{2}{|c|}{} & negative & Positive & \\
\hline \multirow{2}{*}{ CBNAAT } & negative & 172 & 1 & 173 \\
\cline { 2 - 4 } & positive & 21 & 6 & 27 \\
\hline Total & 193 & 7 & 200 \\
\hline
\end{tabular}


Figure 9 Graph comparing efficacy of CBNAAT v/s sputum microscopy

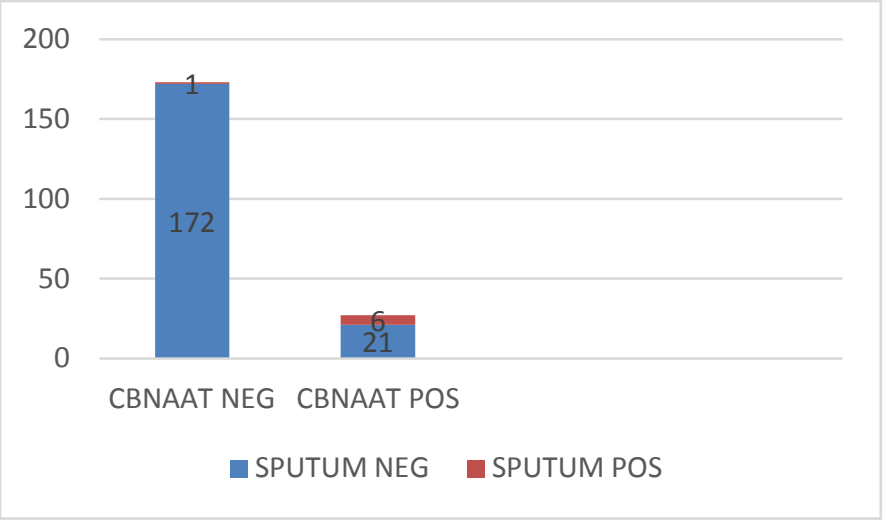

The comparison was significant ( $p$ value $<.001$ ) suggesting an increased efficacy of CBNAAT over sputum microscopy in detecting pulmonary TB.

\section{Comparison between CBNAAT and CD4 count}

Table 11- Comparison between CBNAAT and CD4 count

\begin{tabular}{|ll|c|c|c|}
\hline \multirow{2}{*}{} & & \multicolumn{2}{|c}{ CD4 COUNT } & \multirow{2}{*}{ Total } \\
\cline { 3 - 4 } & & 1 To 200 & 201 To 2000 & \\
\hline \multirow{2}{*}{ Cbnaat } & negative & 25 & 148 & 173 \\
& positive & 19 & 8 & 27 \\
Total & & 44 & 156 & 200 \\
\hline
\end{tabular}

Figure 10 Graph showing comparison between CBNAAT and CD4 count

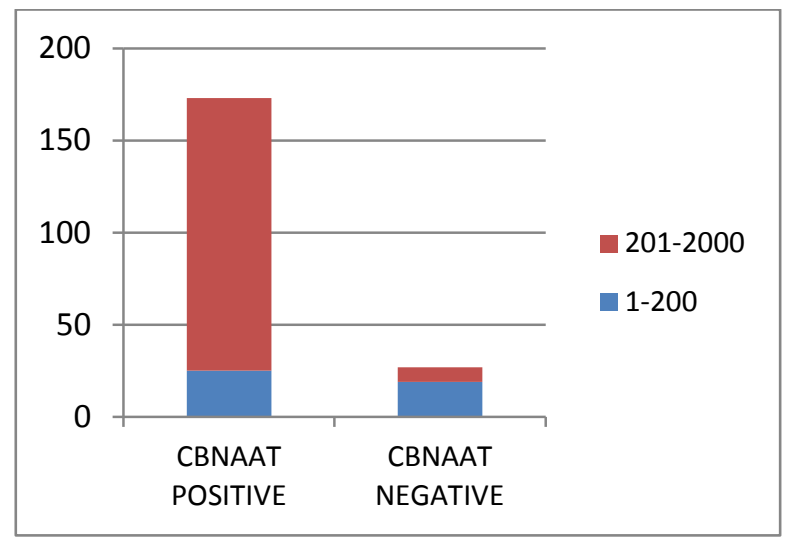

The comparison was significant ( $\mathrm{p}$ value $<0.001$ ) suggesting an increase in CBNAAT positivity with low CD4 count
Comparison between sputum microscopy and CD4 count

Table 12 Comparison between sputum microscopy and CD4 count

\begin{tabular}{|lc|c|c|c|}
\hline & & \multicolumn{2}{|c|}{ CD4 COUNT MOD } & \multirow{2}{*}{ Total } \\
\cline { 3 - 4 } & & 1 To 200 & 201 To 2000 & \\
\hline sputum & negative & 41 & 152 & 193 \\
microscopy & positive & 3 & 4 & 7 \\
Total & 44 & 156 & 200 \\
\hline
\end{tabular}

Figure 11-Graph comparing distribution of sputum microscopy and CD4 count

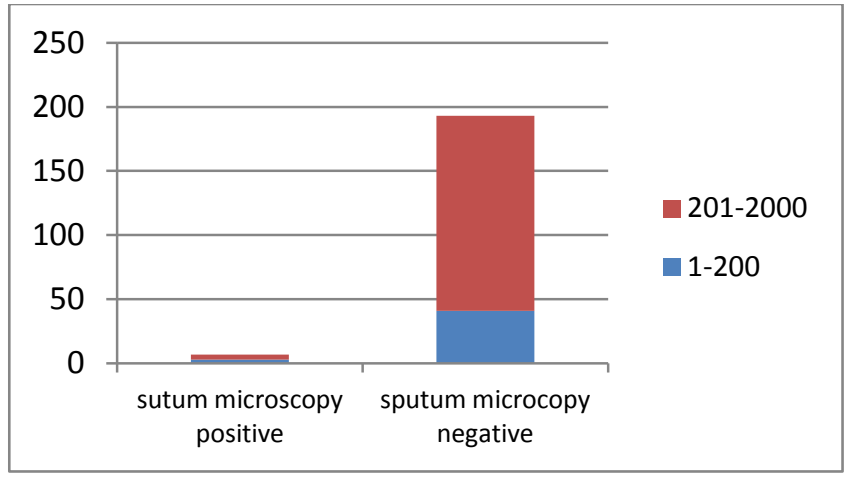

The statistics was not significant ( $\mathrm{p}$ value $>0.180$ ) suggesting lack of correlation between sputum microscopy and CD4 count

\section{Discussion}

This study was primarily undertaken to study the diagnostic accuracy of Xpert MTB/RIF assay at our centre, and compare with the conventional sputum microscopy.

\section{The study group}

Our study group comprised of 200 PLHIV who had at least one symptom out of the 4 symptom complex (current cough, fever, night sweats and weight loss) used for Intensified Case Finding in the group. There was a slight male preponderance of $59 \%$ while $41 \%$ were females. Most other studies have reported greater percentages of males- $79 \%^{(3)}$ and $73 \%^{(4)}$. An exception was the study by Lawn SD et al (5) which showed a female predominance of $65.4 \%$.

This study included adults over 18 years of age and the mean age of the study group was39.4 years. This was slightly on the higher side as compared to other studies which had mean age 
of 37.4 years $^{(3)}$ and 35 years $^{(4)}$. The mean CD4 count of our study population was 396 cells $/ \mathrm{mm}^{3}$. This was significantly higher than other study populations which had CD4 count less than 150 cells $/ \mathrm{mm}^{3^{(6)},(7)}$. Thus, the degree of immunosuppression was lower in our study group. Ours was a cross-sectional study with convenient sampling and we included patients with confirmed HIV positive status and presumptive TB (positive 4 symptom screening). Balcells et al ${ }^{(3)}$, Carriquiry et $\mathrm{al}^{(7)}$ consecutively enrolled adult patients (age $>18$ years) with confirmed HIV infection and suspicion of PTB and the eligible patients had to fulfil at least one of the following inclusion criteria: cough (>10 days), bloody sputum, pneumonia unresponsive to previous antibiotics, fever (>10 days), abnormal CXR or weight

Importantly in this study, the single Xpert outperforms the sputum smear microscopy. This confirms findings from earlier evaluation studies conducted in different settings and corroborates the WHO guidance on replacing smear microscopy with Xpert as the primary diagnostic tool for active TB among HIV-infected individuals.

\section{CBNAAT v/s Sputum Microscopy}

Gene Xpert was positive in 27 cases. Sputum microscopy was positive in 7 cases. Out of 27 CBNAAT positive cases sputum microscopy was positive only in 6 cases. Whereas CBNAAT was negative only in one case of sputum microscopy positivity. Thus CBNAAT was found to be more efficient than sputum microscopy. All these finding were statistically significant ( $p$ value $<0.001)$. Similarly there was a positive correlation between CBNAAT and low CD4 count.CBNAAT sensitivity was more with CD4 count $<200 /$ ml which was statistically significant (p value <0.001). But a study conducted by R.Diwan ${ }^{(8)}$ et al did not show correlation between CBNAAT and CD4 count However such a significant correlation could not be obtained between sputum microscopy and CD4 count.(p value 0.180 ).

\section{Rifampicin Resistance}

Out of the 27 samples which were positive by the Xpert MTB/RIF assay, 3 showed resistance to Rifampicin, an incidence of $11 \%$. It is much less than another Indian study conducted by Isaakidis et $\mathrm{al}^{(9)}$ evaluated the prevalence of Drug Resistant TB HIV-Infected Patients in Mumbai, India. Overall DR-TB was diagnosed in $68(34 \%, 95 \%$ CI: $27 \%-40 \%)$ TB-patients.

We were unable to confirm the rifampicin resistance by other methods But as per other studies, specificity and negative predictive value of Xpert MTB/RIF assay for detection of Rifampicin resistance is around $100 \%{ }^{(10)}$. Previous studies have addressed the issue of false positive rifampicin resistance- Carriquiry et al ${ }^{(3)}$ reported three such cases. WHO has addressed this issue and recommends that rifampicin resistance results of MTB/RIF should be confirmed with further tests and treatment regimens should be based on the latter.

HIV-associated MDR-TB carries a high mortality risk and nosocomial outbreaks in HIV care and treatment centres pose a serious threat to patients accessing these services. Many patients with HIVassociated MDR-TB die before a diagnosis can be made. In this study, the Xpert MTB/RIF assay identified 3 patients with rifampicin resistant isolate who had MDR-TB, greatly reducing the mean time to detection (1 day) compared to using culture- based susceptibility testing (40 days) or using line probe assays on culture isolates (20 days). By accelerating diagnosis of MDR-TB, the Xpert MTB/RIF assay has the potential to substantially reduce the risks of nosocomial transmission of MDR-TB and improve the prognosis of affected individuals ${ }^{(5)}$.

The findings of our study indicate that in persons infected with HIV, Xpert MTB/RIF is a good rule-in test but may have limited rule-out value compared with persons uninfected with HIV. This may be caused by the lower concentration of mycobacteria in the sputum of persons infected with HIV and possibly reduced specificity caused by occult or subclinical disease. Our data add to 
the limited existing knowledge base about the impact of HIV on Xpert MTB/RIF performance. However, there are several important limitations regarding our data (discussed in detail later) and thus further studies are required to clarify these findings. The added rule-out value of a second test in persons infected with HIV who are smearand Xpert MTB/RIF-negative remains to be determined.

Xpert MTB/RIF outperformed smear microscopy because it diagnosed a significant proportion of smear-negative TB cases, and increased the relative proportion of potentially rapidly diagnosed cases by $60 \%$. The added advantage of Xpert over microscopy is that the results of Rifampicin sensitivity are immediately available.

\section{Limitations of our Study}

1. Culture which is the gold standard for diagnosing tuberculosis, was not done.

2. The sample size was small to detect prevalence of TB and diagnostic accuracy of the tests.

3. Follow up of cases could not be done. This was essential in cases of the Xpert positive patients to know if they were responding to ATT and thereby to establish if these cases were actually "true-positives" Sampling was done by convenient sampling

\section{Conclusion}

In conclusion, when used as a routine screening test among people living with HIV, rapid screening using the Xpert MTB/RIF assay substantially increased case detection, supporting replacement of microscopy as the initial diagnostic tool. The assay also greatly decreased the time to diagnosis of tuberculosis and aids in early identification of Rifampicin-resistant tuberculosis.

\section{Bibliography}

1. Jagielski T, Augustynowicz-Kopeć E, Zwolska Z. Epidemiologia gruźlicy w perspektywie świata, Europy i Polski [Epidemiology of tuberculosis: a global, European and Polish perspective]. Wiad Lek. 2010;63(3):230-46. Polish. PMID: 21125747.

2. Facing the Reality of Drug-Resistant Tuberculosis in India: Challenges and Potential Solutions: Summary of a Joint Workshop by the Institute of Medicine, the Indian National Science Academy, and the Indian Council of Medical Research. Washington (DC): National Academies Press (US); 2012. 2, Drug-Resistant TB in India. Available from: https://www.ncbi.nlm.nih.gov/books/NBK 100386/

3. Hanrahan CF, Selibas K, Deery CB, Dansey H, Clouse K, Bassett J, et al. Time to Treatment and Patient Outcomes among TB Suspects Screened by a Single Pointof-Care Xpert MTB/RIF at a Primary Care Clinic in Johannesburg, South Africa. PLoS One. 2013;8(6):e65421. Available from:

http://dx.plos.org/10.1371/journal.pone.00 65421

4. Lawn SD, Brooks S V., Kranzer K, Nicol MP, Whitelaw A, Vogt M, et al. Screening for HIV-Associated Tuberculosis and Rifampicin Resistance before Antiretroviral Therapy Using the Xpert MTB/RIF Assay: A Prospective Study. PLoS Med. 2011;8(7):e1001067. Available from: http://www.ncbi.nlm.nih.gov/pubmed/218 18180

5. Lawn SD, Kerkhoff AD, Vogt $M$, Ghebrekristos Y, Whitelaw A, Wood R. Characteristics and Early Outcomes of Patients With Xpert MTB/RIF-Negative Pulmonary Tuberculosis Diagnosed During Screening Before Antiretroviral Therapy. Clin Infect Dis. 2012;54(8): 1071-9. Available from: 
http://www.ncbi.nlm.nih.gov/pubmed/223 18975

6. Balcells ME, García P, Chanqueo L, Bahamondes L, Lasso M, Gallardo AM, et al. Rapid molecular detection of pulmonary tuberculosis in HIVinfected patients in Santiago, Chile. Int $\mathbf{J}$ Tuberc Lung Dis. 2012;16(10):1349-53. Available from: http://openurl.ingenta.com/content/xref?ge nre $=$ article $\&$ issn $=10273719 \&$ volume $=16$ \&issue $=10 \&$ spage $=1349$

7. Carriquiry G, Otero L, González-Lagos E, Zamudio C, Sánchez E, Nabeta P, et al. A diagnostic accuracy study of Xpert@MTB/RIF in HIV-positive patients with high clinical suspicion of pulmonary tuberculosis in Lima, Peru. PLoS One. 2012;7(9):e44626. Available from: http://www.ncbi.nlm.nih.gov/pubmed/229 70271

8. R Dewan, S Anuradh, A Khanna, S Garg, $S$ Singla et al. Role of cartridge-based nucleic acid amplification test (CBNAAT) for early diagnosis of pulmonary tuberculosis in HIV, Journal, Indian Academy of Clinical Medicine: 2015; 16(2): 114-7

9. Isaakidis P, Das M, Kumar AMV, Peskett C, Khetarpal M, Bamne A, et al. Alarming levels of drug-resistant tuberculosis in HIV-infected patients in metropolitan Mumbai, India. PLoS One. 2014;9(10):110.

10. Al-Darraji HAA, Razak HA, Ng KP, Altice FL, Kamarulzaman A. The Diagnostic Performance of a Single GeneXpert MTB/RIF Assay in an Intensified Tuberculosis Case Finding Survey among HIV-Infected Prisoners in Malaysia. PLoS One. 2013;8(9):1-10. 